\title{
TRABALHADORES COM DEFICIÊNCIA AUDITIVA: RELAÇÕES ENTRE VULNERABILIDADE AO ESTRESSE E SATISFAÇÃO NO TRABALHO
}

Rodolfo A. M. Ambiel

Universidade São Francisco

\author{
Acácia Aparecida Angeli dos Santos \\ Universidade São Francisco
}

\author{
Andressa Cantone de Sousa \\ Universidade São Francisco
}

\begin{abstract}
Resumo
O presente trabalho teve como objetivo explorar as relações entre vulnerabilidade ao estresse laboral e satisfação no trabalho em uma amostra de pessoas com deficiência auditiva. Participaram 54 pessoas, todos trabalhadores, com idades entre 18 e 58 anos $(M=30,37)$. Foram aplicadas a Escala de Vulnerabilidade ao Estresse no Trabalho (EVENT) e a Escala de Satisfação no Trabalho (EST), sendo que o processo de aplicação contou com uma pesquisadora que se comunica por LIBRAS. Os resultados apontaram correlações negativas baixas e moderadas entre os instrumentos. Os homens tiveram maiores escores na EVENT e as mulheres, na EST. Pessoas que falam LIBRAS tiveram médias maiores na EST, enquanto que os demais pontuaram mais na EVENT. Verificou-se que, quanto maior o tempo na mesma empresa, maior a tendência a ser mais vulnerável e menos satisfeito. Conclui-se que novos estudos devem ser feitos, avaliando-se outras variáveis imbricadas no processo de inclusão de pessoas com deficiência auditiva no mercado de trabalho.
\end{abstract}

Palavras-chave: vulnerabilidade ao estresse, satisfação no trabalho, pessoas com deficiência, deficiência auditiva, testes psicológicos.

\section{WORKERS WITH HEARING IMPAIRMENT: RELATIONSHIP BETWEEN VULNERABILITY TO STRESS AND JOB SATISFACTION}

\begin{abstract}
This study aimed to explore the relationships between vulnerability to labour stress and job satisfaction in a sample of people with hearing loss. 54 people were attended, all workers aged between 18 and 58 years $(M=30.37)$. Escala de Vulnerabilidade ao Estresse Laboral (EVENT) and Escala de Satisfação no Trabalho (EST) were applied, and the application process involved a researcher who communicates by Brazilian Signs Language (LIBRAS). The results showed low and moderate negative correlations between the tests. Men had higher scores on the EVENT and the women in the EST. People who speak LIBRAS had the highest averages in the EST, while the others scored more on EVENT. It was found that the longer in the same company, the greater the tendency to be more vulnerable and less satisfied. It is concluded that further studies should be done in order to evaluate other variables intertwined in the process of inclusion of people with hearing loss.

Keywords: vulnerability to stress, job satisfaction, people with disabilities, hearing, psychological tests.
\end{abstract}




\title{
TRABAJADORES CON PÉRDIDA DE LA AUDICIÓN: RELACIONES ENTRE LA VULNERABILIDAD AL ESTRÉS Y LA SATISFACCIÓN EN EL TRABAJO
}

\begin{abstract}
Resumen
Este estudio tuvo como objetivo explorar la relación entre la vulnerabilidad al estrés en el trabajo y la satisfacción laboral en una muestra de personas con discapacidad auditiva. 54 personas participaron, todos trabajadores, entre 18 y 58 años ( $M=$ 30.37). Se aplicó la Escala de Vulnerabilidade ao Estresse no Trabalho (EVENT) y la Escala de Satisfação no Trabalho (EST), y la cosecha de datos ocurrió por intermédio de una investigadora que se comunica por Lenguage Brasileña de Señales (LIBRAS). Los resultados mostraron correlaciones negativas bajas y moderadas entre los instrumentos. Los hombres tuvieron un puntuación mayor en le EVENT y mujeres en le EST. Las personas que hablan por LIBRAS tuvieron promedios más altos en le EST, mientras que los otros tuvieron mejores resultados en le EVENT. Se observó quanto más tempo como trabajador em la empresa, mayor es la tendencia a ser más vulnerable y menos satisfecho. Llegamos a la conclusión de que otros estudios deben realizarse, para evaluar otras variables interrelacionadas en el proceso de inclusión de las personas con discapacidad auditiva en el mercado laboral.

Palabras clave: vulnerabilidad al estrés, satisfacción en el trabajo, personas con discapacidad, discapacidad auditiva, pruebas psicológicas.
\end{abstract}

\section{INTRODUÇÃO}

O Conselho Nacional dos Direitos da Pessoa com Deficiência, pelo Decreto 5.296/04, define deficiência como toda perda ou anormalidade de uma estrutura ou função psicológica, fisiológica ou anatômica que resulte em incapacidade ou altere o desempenho de atividades em geral. Apesar do reconhecimento das perdas em desempenho que pessoas com deficiência podem apresentar, sabe-se também que esse fato, na maioria dos casos, não representa uma limitação ao direito ao trabalho, embora adaptações e adequações, tanto nos ambientes quanto nas relações de trabalho, sejam necessárias, tais como utilização de sinalização luminosa/sonora e a comunicação por meio de leitura e escrita, entre outras (Nambu, 2003; Carvalho-Freitas, 2009)

Segundo Assis, Pussoli e Lacerda (1994), o Brasil é um dos países mais avançados na criação de leis que asseguram o direito ao trabalho para pessoas com deficiência. Um exemplo foi a promulgação da Lei n. ${ }^{\circ} 8.213$, de 25 de julho de 1991, também conhecida como Lei de Cotas, que obriga empresas, com 100 ou mais empregados, a preencher de $2 \%$ a $5 \%$ dos seus cargos com beneficiários reabilitados ou pessoas com deficiências, na seguinte proporção: com até 200 empregados a porcentagem é de 2\%; de 201 a 500 é de 3\%; de 501 a 1000 é de 4\%; e acima de 1001 é de 5\%. 
Antes dessa legislação havia muita dificuldade para ingresso no mercado de trabalho e isso ocorria devido à falta de preparo, tanto das empresas quanto das pessoas com deficiência (Sassaki,1997). Especificamente com relação à pessoa com deficiência auditiva, Klein (1998) refere-se à tendência de uma visão mais integradora, que tem gradativamente substituído o foco em aspectos patológicos das deficiências, passando a considerar o indivíduo com deficiência em toda a sua dimensão. No mesmo sentido, Omote (1994) afirma que, para além do sujeito, a deficiência deve ser compreendida como algo presente na sociedade, e não como algo anômalo. Dessa forma, o trabalho tem se constituído como oportunidade de superação aos estigmas das deficiências, auxiliando na conquista da autonomia e integração à sociedade (Freeman, Carbim \& Boese, 1999).

O perfil de desempenho dessas pessoas no mercado de trabalho é revelado por pesquisadores como Borges, Bello e Leite (2002) que investigaram as razões que levaram cinco empresas de áreas diferentes a contratar pessoas com deficiência auditiva e analisaram a percepção que supervisores e gerentes têm sobre a eficiência delas, quando comparadas com a de colegas ouvintes. Os resultados mostraram que $40 \%$ contratavam pessoas com deficiência para cumprir a lei, outros $40 \%$ revelavam que se beneficiavam aceitando os deficientes auditivos com custo menor; 20\% deram respostas diversificadas. Quanto à diferença de percepção de desempenho no trabalho, os autores verificaram que no item rapidez, $40 \%$ dos entrevistados consideravam as pessoas com deficiência auditiva mais rápidas, enquanto $60 \%$ afirmavam não haver diferença. Com respeito à concentração, $60 \%$ viam a pessoa com deficiência auditiva com melhor desempenho, contra $40 \%$ que não percebiam diferença. Quanto à produtividade, 60\% acreditavam que aqueles com deficiência auditiva são mais produtivos, enquanto $40 \%$ não detectavam diferenças entre os grupos.

Entretanto, há muitas dificuldades que se impõem especialmente no que se refere à capacitação dessas pessoas para o trabalho. No estudo de Araujo e Schmidt (2006) foi realizado um levantamento em 30 empresas com mais de 100 funcionários para identificar as dificuldades encontradas no processo de inclusão de pessoas com deficiência diversas. Os resultados apontaram como principal motivo que leva as empresas a não cumprirem a Lei de Cotas é a baixa escolarização e qualificação profissional dessas pessoas. Em razão disso, 
instituições responsáveis pela formação e capacitação para o trabalho ampliaram o foco dos programas para uma formação mais ampla, indo além da mera aprendizagem de função. Em acréscimo, o estudo de Violante e Leite (2011) revela que a desinformação a respeito das pessoas com deficiência, por parte dos empregadores, também se constitui como obstáculo para inclusão.

Apesar das dificuldades, é cada vez maior o número de trabalhadores com deficiência. Por um lado, ao serem incluídas no mundo do trabalho, essas pessoas podem se beneficiar das experiências para seu crescimento e satisfação pessoal e profissional, mas, por outro, ficam também sujeitas às condições próprias do mercado, menos propícias ao desenvolvimento saudável (Araújo, 2005). No que diz respeito às pessoas com surdez a aprendizagem da linguagem por Libras parecer essencial como facilitadora da inserção no mundo do trabalho (Felipe, 2006).

Apesar da constatação de Violante e Leite (2011) de que há uma tendência das empresas em contratar pessoas com deficiência auditiva, não foram encontrados estudos que ajudassem a compreender as experiências dessas pessoas com respeito ao trabalho. Sobre esse aspecto, Mendes, Nunes, Ferreira e Silveira (2004) informam que o principal foco de estudos científicos sobre inclusão de pessoas com deficiência no mercado de trabalho recai sobre a deficiência intelectual, havendo um hiato nos conhecimentos acerca de como ocorre a inclusão de pessoas com outras deficiências. Sem a pretensão de esgotar o assunto, mas como tentativa de contribuir para o conhecimento do fenômeno, o objetivo geral deste artigo é explorar as relações entre vulnerabilidade ao estresse laboral e a satisfação no trabalho em pessoas com deficiência auditiva. Vale destacar que os construtos aqui focalizados são considerados importantes sob a perspectiva do trabalhador, motivo pelo qual estudos sobre eles serão aqui recuperados.

Sobre o termo estresse, as primeiras referências na literatura psicológica foram à palavra inglesa stress, fazendo alusão a um conceito próprio das ciências dos materiais, significando o impacto e as consequências sofridos pelos metais em condições extremas. Na psicologia, o termo ganhou destaque com os estudos de Selye (1936, 1956, 1982), que o vinculou às consequências de demandas que exigem mais do que o próprio corpo permite, tendo como resultado condições fisiológicas e cognitivas desconfortáveis. 
Mais recentemente estudiosos têm se debruçado sobre questões de precarização das condições de trabalho (Franco, Druck \& Seligmann-Silva, 2010). Na psicologia, algumas pesquisas têm sido propostas com o objetivo específico de analisar quais são potenciais fatores estressantes no ambiente de trabalho, considerando que a vulnerabilidade a eles pode favorecer a manifestação de sintomas de estresse (Alves, Chor, Faerstein, Lopes \& Werneck, 2004; Sisto, Baptista, Noronha \& Santos, 2007).

Segundo Arantes e Vieira (2003), os profissionais têm vivido sob contínua tensão no ambiente de trabalho, pois lidam com diversas demandas além das habituais responsabilidades ocupacionais, como a competitividade dentro das empresas e a exigência de aprendizado e aprimoramento contínuos. Dessa forma, uma classe específica de estresse tende a se compor, denominada de estresse laboral. Segundo Paschoal e Tamayo (2005), ele pode ser definido como a sobrecarga, mesmo que apenas subjetiva, de trabalho que o indivíduo enfrenta, provocando reações negativas, ou seja, respostas fisiológicas, psicológicas e comportamentais. No ambiente organizacional, os trabalhadores podem ser mais ou menos suscetíveis a determinados tipos de estímulos estressores.

A percepção dos sujeitos quanto à sensibilidade aos elementos estressores no local de trabalho será aqui entendida como vulnerabilidade ao estresse laboral. Assumindo esse pressuposto, Sisto e cols. (2007) consideram que esse construto é passível de mensuração, ponderando que o ambiente de trabalho contém elementos potencialmente estressores.

Há disponível para o uso profissional dos psicólogos brasileiros uma escala que avalia esse construto, a Escala de Vulnerabilidade ao Estresse no Trabalho (EVENT - Sisto \& cols., 2007), e maior detalhamento sobre ela pode ser encontrado na seção Instrumentos no presente estudo. Contudo, vale salientar que artigos publicados no Brasil têm usado a escala e mostrado resultados que apontam para a adequação do instrumento pela associação de seus resultados de medidas de construtos similares e com outros relacionados. Cita-se no primeiro caso, o trabalho de Miguel e Noronha (2007) que correlacionaram a pontuação da EVENT com um inventário de sintomas de estresse. Em relação ao segundo caso, foram publicados trabalhos que ilustraram de sua correlação com um teste de raciocínio (Noronha \& Fernandes, 2007) e com uma medida de atenção concentrada (Baptista, Rueda \& Sisto, 2007). Dessa forma, tais 
pesquisas ampliaram as suas evidências de validade no que se refere à relação dessa medida com outros construtos.

O segundo conceito focalizado neste artigo refere-se à satisfação no trabalho. Locke (1976) definiu satisfação no trabalho como um estado emocional agradável ou positivo, que resulta de algum trabalho ou de experiência no trabalho, definição que tem, ainda hoje, o impacto mais importante. Segundo O'Reilly (1990) esse foi um dos temas mais estudados no Século XX e segundo Taylor (1974), nas primeiras décadas, ele abarcava aspectos como fadiga e o salário como influências para a satisfação e produtividade.

Martins (1984), baseada na definição de Locke, afirma que o homem usa de sua bagagem individual de crenças e valores para avaliar seu trabalho e essa avaliação resulta em um estado emocional que, se for agradável, produz satisfação, e, se for desagradável, leva à insatisfação. Portanto, satisfação no trabalho é uma variável de natureza afetiva e se constitui em um processo mental de avaliação das experiências no trabalho que resulta em um estado agradável ou desagradável.

No Brasil, ao menos duas medidas para avaliação no contexto laboral já foram estudadas e constam nos trabalhos de Siqueira (1995; 2008) e Martins e Santos (2006). Neste último, as autoras propuseram um instrumento que avalia, por meio de 46 itens, seis fatores, que são satisfação com suporte organizacional, satisfação com utilidade social da organização e do trabalho, satisfação com relacionamento afetivo no trabalho, satisfação com reconhecimento profissional, insatisfação com inadequação da chefia e falta de oportunidades e insatisfação com sobrecarga de trabalho, tendo apresentado também bons índices de precisão. Contudo, neste estudo, o instrumento utilizado foi o de Siqueira (2008), que avalia cinco fatores com cinco itens em cada um. Maiores informações sobre o instrumento podem ser encontrados na seção Instrumentos. A seguir, serão relatados alguns estudos a respeito de satisfação no trabalho.

O estudo de Rebouças, Legay e Abelha (2007) teve o objetivo de analisar o nível de satisfação no trabalho e o impacto causado nos profissionais de um serviço de saúde mental e possível associações com variáveis sociodemográficas e funcionais. Foi utilizado um estudo transversal com 321 profissionais de uma instituição de saúde mental de longa permanência na cidade do Rio de Janeiro. Os instrumentos utilizados foram as escalas de avaliação da Satisfação da equipe 
em serviços de saúde mental e um questionário sobre características sociodemográficas e profissionais. Os resultados indicaram que a maioria das características associadas aos menores níveis de satisfação no trabalho foi associada aos mais elevados níveis de improdutividade no trabalho.

Suehiro, Santos, Hatamoto e Cardoso (2008) avaliaram a vulnerabilidade ao estresse e satisfação no trabalho em 55 profissionais de um programa de saúde da família, mulheres, com idades entre 18 e 52 anos. Foram observadas correlações significativas, negativas e moderadas entre vários fatores da EVENT e da EST, com destaque para Clima e funcionamento Organizacional com Satisfação com o Salário e Promoções; Pressão no Trabalho com Salário e Natureza do Trabalho; e Infraestrutura e Rotina com Salário e Natureza do Trabalho. Os resultados indicaram também uma relação positiva entre escolaridade e Satisfação com Natureza do Trabalho.

Ainda relativo à essa temática, Ruviaro e Bardagi (2010) estudaram as relações entre sintomas de síndrome de Burnout (Maslach Burnout InventoryMBI) e Satisfação no Trabalho (EST) em 86 profissionais da área da saúde. Foram verificadas correlações significativas, negativas e fracas entre satisfação com colegas e tempo de serviço, e moderadas entre satisfação com salário, natureza da tarefa, promoções e pontuação total da EST com Exaustão emocional. Esses mesmos fatores da EST também se correlacionaram significativa, positiva e moderadamente com o fator Realização Profissional do MBI.

Tendo em vista o exposto, o presente trabalho se propõe a explorar as relações entre vulnerabilidade ao estresse laboral e satisfação no trabalho em uma amostra de trabalhadores com deficiência auditiva. Em acréscimo, serão verificadas eventuais diferenças relativas a variáveis como sexo, utilização ou não Língua Brasileira de Sinais (LIBRAS), faixa etária e tempo de emprego.

\section{MÉTODO}

\section{Participantes}

A amostra pesquisada foi de conveniência, e participaram dela 54 trabalhadores com deficiência auditiva provenientes de empresas privadas do interior do estado de São Paulo, com idades entre 18 e 58 anos $(M=30,37$; $D P=9,76)$. Destaca-se que 50\% $(n=27)$ concentram-se na idade entre 18 e 27 anos e o tempo médio de trabalho na empresa atual foi de 1,98 anos $(D P=0,92)$. 
Quanto ao cargo, 96,3\% $(n=52)$ estavam em cargos operacionais e apenas $3,7 \%(n=2)$ em cargos administrativos. Do total de participantes $59,3 \%(n=32)$ eram do sexo masculino e $40,7 \%(n=22)$ do sexo feminino, sendo que $61,1 \%$ $(n=33)$ possuíam escolaridade entre ensino médio completo e incompleto e os demais haviam cursado apenas o ensino fundamental. A maioria dos participantes $(72,2 \%)$ comunica-se por LIBRAS e $27,8 \%(n=15)$ usam outros recursos de comunicação, tais como leitura em língua portuguesa e leitura labial.

\section{Instrumentos}

Dois instrumentos usados na presente pesquisa são apresentados a seguir. São destacados em sua descrição informações sobre os parâmetros psicométricos de cada um deles.

Escala de Vulnerabilidade ao Estresse no Trabalho (EVENT) - Sisto, Baptista, Noronha \& Santos, 2007)

Os itens abordam assuntos relacionados ao cotidiano de trabalho e possíveis relações com a vulnerabilidade emocional do funcionário. O teste apresenta 40 questões que avaliam três fatores: Clima e funcionamento organizacional (16 itens); Pressão no trabalho (13 itens); Infraestrutura e rotina (11 itens). As respostas estão dispostas em nunca (0), às vezes (1) e frequentemente (2) e sua amostra normativa foi composta por indivíduos de 17 a 54 anos, que responderam à escala na forma individual e coletiva. Encontra-se atualmente na lista de testes aprovados pelo Conselho Federal de Psicologia (CFP), podendo ser utilizada profissional pelos psicólogos brasileiros.

A principal evidência de validade é a obtida pela análise da estrutura interna dos itens, que, por meio da análise fatorial exploratória, indicou a presença dos três fatores referidos, explicando $37,44 \%$ da variância. Os índices de consistência interna são adequados visto que variaram de 0,77 a 0,91.

Escala de Satisfação no Trabalho (EST) - Siqueira (2008)

As questões propostas estão relacionadas ao cotidiano de trabalho e tem como objetivo avaliar o grau de contentamento do trabalhador frente a cinco dimensões, que são Satisfação com colegas, Satisfação com o salário, Satisfação com a chefia, Satisfação com a natureza do trabalho e Satisfação com as 
promoções. É composto por 25 itens em escala Likert de 7 pontos, ou seja, insatisfeito (1), muito insatisfeito (2), insatisfeito (3), indiferente (4), satisfeito (5), muito satisfeito (6), totalmente satisfeito (7), podendo ser aplicado individual ou coletivamente. Os fatores foram obtidos por análise fatorial, na qual se observou a existência de 5 dimensões com 5 itens cada, explicando $48 \%$ da variância. Os coeficientes Alfa de Cronbach variaram entre 0,84 e 0,91.

No cabeçalho dos instrumentos havia questões que permitiram a identificação de variáveis sociodemográficas. Vale ressaltar que foi incluído mais um tópico referente ao uso da linguagem LIBRAS.

\section{Procedimento}

Inicialmente foi realizado contato com uma instituição que promove a formação de pessoas com deficiência auditiva para o mercado de trabalho, quando os objetivos da pesquisa foram explicados. Tal procedimento se deu com a instituição, uma vez que os participantes eram trabalhadores de diversas empresas, mas que passavam por atendimentos periódicos em tal instituição. Após aprovado pelo Comitê de Ética, os participantes foram contatados e os objetivos da pesquisa Ihes foram explicados. Uma das autoras do presente artigo comunica-se por LIBRAS e utilizou-se dessa linguagem para conversar com aqueles que a entendiam. Com os demais, o processo foi diferenciado, pois a explicação foi feita por escrito e com o auxílio dos profissionais que trabalhavam semanalmente com os participantes. A coleta foi realizada coletivamente em seis sessões com duração aproximada de 1 hora e 30 minutos cada sessão. Esse número de encontros foi necessário não só pela questão do espaço físico, como também porque os participantes foram agrupados em razão de usarem ou não LIBRAS.

\section{Análise de dados}

As escalas foram corrigidas seguindo-se a atribuição de pontuação descrita no Método, tal como recomendada pelos autores dos respectivos instrumentos. $A$ análise estatística escolhida foi a paramétrica, de acordo com teste de normalidade apresentado nos resultados, sendo usadas, além das análises descritivas, o teste t de Student quando comparadas duas variáveis. Quando da comparação de mais de duas variáveis recorreu-se à ANOVA e ao teste post hoc 
de Tukey para identificar os grupos que se diferenciaram. A prova de Pearson foi escolhida para o estabelecimento dos índices de correlação.

\section{RESULTADOS}

Os dados foram organizados em planilha eletrônica (SPSS 17) e apresentados conforme a ordem dos objetivos propostos. Para efeito de melhor compreensão dos resultados são apresentados em primeiro lugar na Tabela 1 as estatísticas descritivas dos instrumentos.

Tabela 1.

Estatística descritiva dos instrumentos aplicados na amostra de surdos $(N=54)$ em cidade do interior de São Paulo no ano de 2007.

\begin{tabular}{ccccc}
\hline EVENT & Mínimo & Máximo & Média & DP \\
\hline Clima e funcionamento organizacional & 16 & 36 & 26,64 & 4,76 \\
Pressão no trabalho & 15 & 36 & 24,61 & 4,05 \\
Infraestrutura e rotina & 11 & 24 & 18,32 & 3,22 \\
Total & 35 & 91 & 68,74 & 11,72 \\
\hline EST & & & & \\
Satisfação com Salário & 13 & 25 & 19,17 & 3,63 \\
Satisfação com Colegas & 13 & 27 & 19,96 & 3,34 \\
Satisfação com Chefia & 14 & 28 & 21,52 & 3,16 \\
Satisfação com Promoções & 10 & 24 & 18,70 & 3,51 \\
Satisfação com Natureza do Trabalho & 15 & 25 & 20,52 & 2,83 \\
Total & 78 & 121 & 99,52 & 11,84 \\
\hline
\end{tabular}

O fator Clima e Funcionamento Organizacional da EVENT tem pontuação máxima possível de 48 pontos e se destacou com média de 26,64 ( $D P=4,75)$. Os resultados da EST que mais de destacaram foram Satisfação com chefia, com média de 21,52 ( $\mathrm{DP}=3,1)$ e Satisfação com Natureza do trabalho com média 20,52 (DP=2,83). Já os fatores Satisfação com Promoções com média 18,70 $(D P=3,51)$ e Satisfação com Salário, com média 19,17 ( $D P=3,62)$ foram os fatores com as médias mais baixas.

Para verificar a normalidade da distribuição das pontuações na amostra estudada, foram verificadas as pontuações totais dos instrumentos. Para a EVENT, a medida de assimetria (skewness) ficou em $-0,873$, enquanto que 0 achatamento (kurtosis) ficou em 1,064. Para a EST, os índices encontrados 
foram de 0,027 de assimetria (skewness) e -0,856 de achatamento (kurtosis). Ao utilizar o teste de normalidade de Kolmogorov-Smirnov, não observou-se diferença significativa em nenhum dos instrumentos (EVENT: $p=0,20 ;$ EST: $p=0,06)$, indicando que a distribuição da amostra deste estudo não se difere de uma distribuição normal. Dessa forma, optou-se por utilizar estatística paramétrica, conforme justificado em "Análise de dados". A seguir, a Tabela 2 apresenta as correlações entre os instrumentos.

Tabela 2.

Correlações entre Escala de Vulnerabilidade ao Estresse no Trabalho e com a Escala de Satisfação no Trabalho.

\begin{tabular}{cccccc} 
Satisfação & $\begin{array}{c}\text { Satisfação } \\
\text { com } \\
\text { Salário }\end{array}$ & $\begin{array}{c}\text { Som } \\
\text { Colegas }\end{array}$ & $\begin{array}{c}\text { Satisfação } \\
\text { com Chefia }\end{array}$ & $\begin{array}{c}\text { Satisfaçãão } \\
\text { com } \\
\text { Promoções }\end{array}$ & $\begin{array}{c}\text { Natureza do } \\
\text { Trabalho }\end{array}$ \\
\hline $\begin{array}{c}\text { Clima e } \\
\text { funcionamento } \\
\text { organizacional } \\
\begin{array}{c}\text { Pressão no } \\
\text { trabalho }\end{array}\end{array}$ & $-0,12$ & $-0,07$ & $-0,37^{* *}$ & 0,09 & $-0,26$ \\
$\begin{array}{c}\text { Infraestrutura e } \\
\text { rotina }\end{array}$ & $-0,22$ & $-0,40^{* *}$ & $-0,36^{* *}$ & $-0,47^{* *}$ & $-0,11$ \\
\hline$*: p<0,01 ; * *: p<0,001$ & $-0,20$ & $-0,45^{* *}$ & $-0,09$ & $-0,33^{*}$ \\
\hline
\end{tabular}

Valendo-se da prova de correlação de Pearson (Tabela 2) foi possível observar que todas as correlações significativas foram negativas. A análise da força da magnitude foi feita com base em Dancey e Reidy (2006). Com relação ao fator Clima e funcionamento organizacional da EVENT, foi observado um índice de correlação baixo com o fator Satisfação com Chefia da EST. Com o fator Pressão no trabalho da EVENT, foram observados um índice de correlação baixa com o fator Satisfação com Chefia e dois deles moderados com a Satisfação com Colegas, e Satisfação com Promoções da EST. Por fim, o fator Infraestrutura e rotina mostrou-se correlacionado negativamente e com magnitude moderada com o fator Satisfação com Chefia e baixa com o fator Satisfação com Natureza do trabalho da EST. A Tabela 3 mostra a análise de diferença de média entre sexos. 
Tabela 3.

Comparação entre sexos.

\begin{tabular}{|c|c|c|c|c|c|c|}
\hline & Sexo & Média & DP & $t$ & $g l$ & $p$ \\
\hline EVENT & & & & & & \\
\hline \multirow{2}{*}{$\begin{array}{c}\text { Clima e } \\
\text { funcionamento } \\
\text { organizacional }\end{array}$} & Mulheres & 25,55 & 4,64 & $-1,427$ & 52 & 0,16 \\
\hline & Homens & 27,42 & 4,76 & & & \\
\hline \multirow{2}{*}{$\begin{array}{l}\text { Pressão no } \\
\text { trabalho }\end{array}$} & Mulheres & 23,55 & 4,06 & $-1,627$ & 52 & 0,11 \\
\hline & Homens & 25,34 & 3,95 & & & \\
\hline \multirow{2}{*}{$\begin{array}{l}\text { Infraestrutura e } \\
\text { rotina }\end{array}$} & Mulheres & 17,29 & 2,61 & $-1,945$ & 52 & 0,06 \\
\hline & Homens & 19,00 & 3,44 & & & \\
\hline \multirow[t]{2}{*}{ Total } & Mulheres & 65,59 & 11,24 & $-1,664$ & 52 & 0,10 \\
\hline & Homens & 70,91 & 11,73 & & & \\
\hline \multicolumn{7}{|l|}{ EST } \\
\hline \multirow[t]{2}{*}{ Salário } & Mulheres & 19,27 & 3,94 & 0,177 & 52 & 0,86 \\
\hline & Homens & 19,09 & 3,46 & & & \\
\hline \multirow[t]{2}{*}{ Colegas } & Mulheres & 20,55 & 2,99 & 1,063 & 52 & 0,29 \\
\hline & Homens & 19,56 & 3,56 & & & \\
\hline \multirow[t]{2}{*}{ Chefia } & Mulheres & 22,59 & 2,72 & 2,136 & 52 & 0,04 \\
\hline & Homens & 20,78 & 3,27 & & & \\
\hline \multirow[t]{2}{*}{ Promoções } & Mulheres & 18,81 & 3,12 & 0,185 & 52 & 0,85 \\
\hline & Homens & 18,63 & 3,79 & & & \\
\hline \multirow[t]{2}{*}{ Natureza } & Mulheres & 21,00 & 2,69 & 1,036 & 52 & 0,31 \\
\hline & Homens & 20,19 & 2,92 & & & \\
\hline \multirow[t]{2}{*}{ Total } & Mulheres & 101,36 & 11,34 & 0,948 & 52 & 0,35 \\
\hline & Homens & 98,25 & 12,19 & & & \\
\hline
\end{tabular}

Foram verificadas diferenças entre sexos em dois fatores, quais sejam, Infraestrutura e Rotina da EVENT e Satisfação com Chefia da EST. Os resultados mostram que os homens são mais vulneráveis ao estresse relacionado à infraestrutura e rotina no ambiente de trabalho do que as mulheres. Já as mulheres se destacam mais que os homens com relação a satisfação com a chefia. A Tabela 4 mostra os resultados da análise de comparação entre pessoas que se comunicam por LIBRAS e aquelas que não utilizam essa linguagem.

Os dados acima descrevem que existe diferença nos fatores Pressão no trabalho da EVENT e Satisfação com Promoção da EST. Os resultados mostram que o grupo de pessoas que não falam LIBRAS é mais vulnerável a pressão no trabalho do que as pessoas que falam libras, enquanto que as pessoas que falam libras apresentam maior satisfação com relação à promoção no trabalho. 
Tabela 4. Comparação entre pessoas que se comunicam por LIBRAS e outros recursos de comunicação.

\begin{tabular}{|c|c|c|c|c|c|c|}
\hline & LIBRAS & Média & DP & $t$ & $g l$ & $p$ \\
\hline \multicolumn{7}{|l|}{ EVENT } \\
\hline \multirow{2}{*}{$\begin{array}{c}\text { Clima e } \\
\text { funcionamento } \\
\text { organizacional }\end{array}$} & Sim & 27,24 & 5,01 & 1,466 & 52 & 0,15 \\
\hline & Não & 25,13 & 3,78 & & & \\
\hline \multirow[t]{2}{*}{ Pressão no trabalho } & Sim & 23,85 & 3,88 & $-2,327$ & 52 & 0,02 \\
\hline & Não & 26,60 & 3,94 & & & \\
\hline \multirow{2}{*}{$\begin{array}{l}\text { Infraestrutura e } \\
\text { rotina }\end{array}$} & Sim & 18,61 & 3,31 & 1,024 & 52 & 0,31 \\
\hline & Não & 17,60 & 2,97 & & & \\
\hline \multirow[t]{2}{*}{ Total } & Sim & 68,51 & 12,72 & $-0,228$ & 52 & 0,82 \\
\hline & Não & 69,33 & 8,97 & & & \\
\hline \multicolumn{7}{|l|}{$E S T$} \\
\hline \multirow[t]{2}{*}{ Salário } & Sim & 19,49 & 3,63 & 1,048 & 52 & 0,30 \\
\hline & Não & 18,33 & 3,62 & & & \\
\hline \multirow[t]{2}{*}{ Colegas } & Sim & 20,26 & 3,35 & 1,041 & 52 & 0,30 \\
\hline & Não & 19,20 & 3,30 & & & \\
\hline \multirow[t]{2}{*}{ Chefia } & Sim & 21,39 & 3,08 & $-0,498$ & 52 & 0,62 \\
\hline & Não & 21,87 & 3,46 & & & \\
\hline \multirow[t]{2}{*}{ Promoções } & Sim & 19,42 & 3,29 & 2,504 & 52 & 0,02 \\
\hline & Não & 16,87 & 3,50 & & & \\
\hline \multirow[t]{2}{*}{ Natureza } & Sim & 20,15 & 2,85 & $-1,545$ & 52 & 0,13 \\
\hline & Não & 21,47 & 2,64 & & & \\
\hline \multirow[t]{2}{*}{ Total } & Sim & 100,21 & 12,28 & 0,684 & 52 & 0,50 \\
\hline & Não & 97,73 & 10,81 & & & \\
\hline
\end{tabular}

Com relação à verificação de diferenças entre faixas etárias, foram realizadas ANOVAS e a amostra foi dividida em quatro grupos. O grupo 1 conta com pessoas de até 23 anos $(n=16)$, o grupo 2 com pessoas de 24 a 27 anos $(n=11)$, o grupo 3 com pessoas de 28 a 36 anos $(n=15)$ e o grupo 4 com pessoas de 37 anos em diante $(n=12)$. As diferenças detectadas podem ser atribuídas ao acaso, não havendo qualquer resultado que tenha sido significativo.

Foram também exploradas eventuais diferenças que poderiam ser atribuídas ao tempo de emprego. Para tanto, os participantes foram divididos em três grupos, a saber, até 3 meses de emprego $(n=19)$, de quatro meses a um ano $(n=20)$ e com mais de um ano de emprego $(n=15)$. 
A partir dessa formulação, foi realizada a ANOVA, indicando que cinco diferenças significativas foram encontradas, Pressão no trabalho $(F(2,52)=6,136$; $p=0,004)$, Infraestrutura e rotina $(F(2,52)=7,876 ; p=0,001)$ e Total da EVENT $(F=(2,52)=4,198 ; p=0,02)$, e Satisfação com Salário $(F(2,52)=4,855 ; p=0,01)$ e com Promoções $(F(2,52)=11,929 ; p=0,000)$ da EST. Visando esclarecer tais diferenças, foram solicitadas análises post hoc de Tukey, que podem ser visualizadas na Tabela 5.

Tabela 5. Prova post hoc de Tukey em relação ao tempo de trabalho.

\begin{tabular}{|c|c|c|c|}
\hline & & \multicolumn{2}{|c|}{ Subconjuntos para Alfa $=0,05$} \\
\hline & & 1 & 2 \\
\hline & Até 3 meses & 22,53 & \\
\hline \multirow[t]{3}{*}{ Pressão no Trabalho } & De 4 meses a 1 ano & 24,80 & 24,80 \\
\hline & Mais que 1 ano & & 27,00 \\
\hline & $\mathrm{p}$ & 0,172 & 0,192 \\
\hline \multirow[t]{4}{*}{ Infraestrutura e Rotina } & Até 3 meses & 16,53 & \\
\hline & Mais que 1 ano & 18,20 & 18,20 \\
\hline & De 4 meses a 1 ano & & 20,21 \\
\hline & $\mathrm{p}$ & 0,206 & 0,106 \\
\hline \multirow[t]{4}{*}{ Total (EVENT) } & Até 3 meses & 62,84 & \\
\hline & Mais que 1 ano & 71,33 & 71,33 \\
\hline & De 4 meses a 1 ano & & 72,40 \\
\hline & $\mathrm{p}$ & 0,07 & 0,956 \\
\hline \multirow[t]{4}{*}{ Salário } & Mais que 1 ano & 17,47 & \\
\hline & De 4 meses a 1 ano & 18,70 & 18,70 \\
\hline & Até 3 meses & & 21,00 \\
\hline & $\mathrm{p}$ & 0,529 & 0,118 \\
\hline \multirow[t]{4}{*}{ Promoções } & Mais que 1 ano & 15,87 & \\
\hline & De 4 meses a 1 ano & & 18,85 \\
\hline & Até 3 meses & & 20,89 \\
\hline & $\mathrm{p}$ & 1,000 & 0,113 \\
\hline
\end{tabular}

Os dados indicam que o grupo com mais tempo de emprego diferenciou-se significativamente quanto a sua percepção de vulnerabilidade a eventos estressores relacionados à pressão no trabalho do grupo que está trabalhando há até três meses. O grupo intermediário, entre quatro meses e um ano de emprego, não se diferenciou em nenhum dos grupos. Para o fator Infraestrutura e Rotina e Total da EVENT, se observou que o grupos com menos tempo de 
emprego diferenciou-se daquele com período de 4 meses a 1 ano no trabalho, sendo maior a pontuação para o último grupo.

A análise post hoc realizada, para o fator Satisfação com o Salário, da EST, mostrou que os grupos com mais e menos tempo de emprego novamente se diferenciaram. Entretanto, com relação à satisfação com o salário, percebe-se que o grupo com menos tempo obteve maior média que o grupo com mais tempo. Com relação ao fator Satisfação com Promoções, da EST, pode-se observar que no primeiro subconjunto, com a menor média, o grupo com mais de um ano de emprego ficou alocado, enquanto os grupos com pessoas até um ano de emprego ficaram no segundo subconjunto, com médias significativamente maiores que o primeiro.

\section{DISCUSSÃO}

O objetivo deste estudo foi de explorar as relações entre vulnerabilidade ao estresse laboral e satisfação no trabalho em uma amostra de trabalhadores com deficiência auditiva. Para além da correlação entre os instrumentos, buscou-se também verificar se os dados teriam alguma variação quando comparados em relação às variáveis sexo, comunicação por LIBRAS, faixa etária e tempo no emprego.

Os resultados evidenciaram que, tal qual o estudo de Suehiro e cols. (2008), as correlações significativas encontradas entre vulnerabilidade ao estresse e satisfação no trabalho foram negativas, sendo detectadas diferenças nas magnitudes das mesmas, comentadas posteriormente. Da mesma forma, o estudo de Ruviaro e Bardagi (2010) também encontraram relações negativas entre satisfação no trabalho e exaustão emocional, como uma das dimensões do estresse. Ao considerar os fundamentos teóricos dos construtos, pode-se afirmar que as relações negativas seriam esperadas previamente por se tratarem de condições com conotações afetivas opostas em relação às experiências vivenciadas no ambiente do trabalho (Sisto \& cols, 2007; Locke, 1976; Martins, 1984).

Especificamente quanto à associação entre os fatores dos instrumentos utilizados neste estudo, observa-se que quanto mais os participantes se perceberam como vulneráveis ao estresse em relação ao clima e funcionamento da empresa, os mesmo mostraram-se menos satisfeito com a chefia. Este resultado sugere que, para a amostra deste estudo, as relações com superiores 
parecem ser importantes fontes de estresse, sendo que aos chefes parece ser atribuída uma importância em relação ao bom clima e funcionamento no ambiente de trabalho. Este fato merece receber especial atenção ao se constatar que em todos os fatores da EVENT foram observadas correlações negativas com satisfação com chefia.

Tal resultado difere do encontrado por Suehiro e cols. (2008), cujos dados coletados junto a mulheres profissionais de programa saúde da família revelaram relação negativa entre Clima e Funcionamento Organizacional da EVENT com Satisfação com Salário e com Promoções, da EST. Possivelmente, as diferenças entre as amostras se deva às peculiaridades as funções, uma vez que os trabalhadores com deficiência auditiva que compuseram esta amostra trabalham em serviços internos, sejam operacionais ou administrativos, nos quais tendem a ficar mais frequentemente sob supervisão direta de chefes, o que pode ter modulado suas percepções nesse sentido.

Dentre as outras correlações encontradas, percebe-se que a única que foi coincidente com o estudo de Suehiro e cols. (2008) foi entre o fator Infraestrutura e rotina da EVENT com Natureza do Trabalho (EST). Assim, considerando que as funções e atividades típicas de determinado emprego estão diretamente subordinadas à infraestrutura disponível para realizá-las bem como à rotina de tarefas e horários imposta pela empresa para sua consumação, podese considerar que, independente de qual seja, a natureza do trabalho pode-se impor com uma importante fonte de estressores.

Quantos às comparações entre grupos um primeiro resultado que merece destaque é relativo à análise de diferença de médias entre os sexos, sendo que os homens se mostraram mais vulneráveis ao estresse no fator Infraestrutura e Rotina e as mulheres tiveram maiores médias em Satisfação com a chefia. Com relação à EVENT, o estudo de Noronha e Fernandes (2007) encontrou resultados diferentes, sendo que as mulheres obtiveram maiores médias em Infraestrutura e rotina, enquanto que Miguel e Noronha (2007) não encontraram diferenças significativas. Esses dados conduzem à reflexão no sentido de que, independente da amostra, homens e mulheres parecem não mostrar uma padrão contínuo de vulnerabilidade ao estresse, especialmente no que toca ao fator Infraestrutura e Rotina. Uma hipótese é que a vulnerabilidade, sendo uma variável pessoal, seja dependente do ambiente laboral em que as pessoas estão inseridas. Nesse sentido, possivelmente, ambientes com mais elementos estressores podem 
potencializar a percepção da vulnerabilidade. Quanto à satisfação com a chefia, é interessante notar que, conforme já discutido, este foi o único fator da EST relacionado a todos os fatores da EVENT. Assim, ao se evidenciar que mulheres apresentaram significativamente maiores médias do que os homens neste fator, é necessário lembrar que elas foram minoria na composição da amostra e, ao apresentar um padrão diferenciado de relacionamento com a chefia, pode-se hipotetizar que os índices de correlação negativos que foram encontrados possam ter ocorrido de forma tão marcada devido ao padrão masculino de respostas ao EST.

Outro foco desta pesquisa foi o de verificar as diferenças entre as percepções de pessoas que se comunicam ou não por LIBRAS, partindo do princípio que a comunicação é um fator preponderante nas relações de trabalho. Foram verificadas diferenças em Pressão no trabalho (EVENT) e Satisfação com Promoções e, a se julgar pelos resultados, o fato de se comunicar por LIBRAS parece ter uma influência importante no contexto de trabalho, uma vez que aquele que se comunicam tiveram maior média em satisfação e os que não se comunicam dessa forma sobressaíram em vulnerabilidade ao estresse. Esse dado, embora necessite de mais informações, parece ser interessante para pessoas com deficiências auditivas e suas famílias, bem como para as empresas, no sentido de se investir em capacitação em LIBRAS como forma de proporcionar melhor qualidade de desempenho e satisfação no trabalho.

Os resultados sobre o tempo no emprego indicaram que pessoas com até três meses de emprego tiveram menores médias em dois fatores (Pressão no trabalho e Infraestrutura e Rotina) e no escore geral da EVENT, bem como maiores médias em dois fatores da EST (Salário e Promoções), sendo que, em satisfação, as menores médias, em ambos os fatores, foram alcançadas pelo grupo com mais de um ano. Além disso, é importante relembrar também não houve diferença significativa entre as faixas etárias dos participantes.

Em face desses resultados, pode-se pensar que, ao longo do processo de adaptação ao ambiente e às tarefas do trabalho, as pessoas tendem a experimentar mudanças quantos às relações e às demandas, tanto em relação à exigência quanto à rotina das tarefas. Por sua vez, o aumento das demandas podem gerar expectativas de possíveis melhorias em relação ao cargo e ao salário. 
Dessa forma, considerando que a satisfação laboral é uma variável afetiva, fruto de uma avaliação cognitiva de experiências de trabalho (Martins, 1984), quando o aumento da demanda é avaliado como sobrecarga e as expectativas de recompensa não são atendidas com o passar do tempo (Paschoal \& Tamayo, 2005), tal avaliação pode se tornar negativa e o trabalhador tende a ficar mais vulnerável a determinados eventos, gerando um estado afetivo desagradável e podendo conduzir a improdutividade (Rebouças \& cols., 2007). A questão da inclusão social e no trabalho de pessoas com deficiência ainda é recente e pouco explorada como tema de pesquisa, apesar de alguns avanços (Assis \& cols., 1994; Freeman \& cols., 1999). Assim, a oportunidade de trabalhar pode ser algo valorizado ao ponto de, inicialmente, ser mais forte do que as situações estressantes e percepções de insatisfação.

\section{CONSIDERAÇÕES FINAIS}

Pesquisas desta natureza são necessárias, já que a compreensão da empresa e do colaborador sobre esse tema ainda é incipiente. Assim, o presente estudo pode contribuir para a orientação de pessoas com deficiências auditivas no ambiente de trabalho bem como para empregadores e profissionais de recursos humanos, uma vez que aborda um assunto ainda pouco explorado na literatura científica.

Salienta-se como limitações do presente estudo o pequeno número de participantes e o fato de todos serem oriundos de uma mesma localidade. Ao lado disso, a coleta de dados foi pontual, retratando a percepção dos indivíduos em um dado momento. Para tanto, amostras maiores e diversificadas em relação à focalizada neste artigo se fazem necessárias, bem como a realização de estudos longitudinais que acompanhem os trabalhadores que entraram no mercado de trabalho na cota de inclusão. Além disso, os instrumentos escolhidos para a realização deste estudo não haviam ainda sido testados em amostras de pessoas com deficiência visual, o que possivelmente fez com que aspectos importantes tenham deixado de ser abordados, tais como elementos potencialmente estressores e/ou geradores de insatisfação, como a falta de conhecimento e informação dos empregadores acerca das necessidades específicas para esse público. Em estudos futuros, pode-se pensar em instrumentos contendo itens específicos para o público com deficiência. Também 
pesquisas utilizando a entrevista como meio de coleta de dados poderão contribuir para um maior conhecimento sobres os aspectos aqui abordados.

Por fim, deve-se ressaltar a importância de que no campo da avaliação psicológica, outros instrumentos sejam estudados, incluindo amostras específicas e diferentes daquelas para os quais há estudos normativos com amostras de pessoas sem deficiências declaradas. Sabe-se que muitos psicólogos usam recursos de adaptação, sem o respaldo de dados empíricos sobre a validade das interpretações obtidas em pessoas com deficiências específicas. Dados obtidos com estudos como esses, poderiam ampliar o uso de testes no rol de instrumentos adotados nos processos seletivos e organizacionais, desde que fossem adaptados à realidade de inclusão de pessoas com deficiência no mercado de trabalho.

\section{REFERÊNCIAS}

Alves, M. G. M., Chor, D., Faerstein E, Lopes, C. S., \& Werneck, G. L. (2004). Versão resumida da "job stress scale": Adaptação para o português. Revista de Saúde Publica, 38(2),164-171.

Arantes, M. A. A. C. \& Vieira, M. J. F. (2003). Estresse (2a ed.). São Paulo: Casa do Psicólogo.

Araújo, M. A. N. (2005). A estruturação da linguagem e a formação de conceitos na qualificação de surdos para o trabalho. Psicologia: Ciência e Profissão, 25(2), 240-25.

Araujo, J. P. \& Schmidt, A. (2006). A inclusão de pessoas com necessidades especiais no trabalho: A visão de empresas e de instituições educacionais especiais na cidade de Curitiba. Revista Brasileira de Educação Especial, 12(2), p.241-254.

Assis, O. Q., Pussoli, L. \& Lacerda, V. A. (1994). Os direitos da pessoa portadora de deficiência. São Paulo: Lúmen.

Baptista, M. N., Rueda, F. J. M., \& Sisto, F. F. (2007). Relação entre estresse laboral e atenção concentrada. Encontro Revista de Psicologia, 11(16), 7589.

Borges, L., Bello, R. \& Leite, S. (2002). O deficiente auditivo e o mercado de trabalho. Revista de Ciências Médicas e Biológicas, 1(1), 99-104.

Carlotto, M. S. \& Camara, S. G. (2008). Analise de produção cientifica sobre a síndrome de Burnout no Brasil. Psico, 39(2), 152-158. 
Carvalho-Freitas, M. N. (2009). Ações de adequação das condições e práticas de trabalho para inserção de pessoas com deficiência. Psicologia para a América Latina, 16. Recuperado em 04 de outubro de 2011, de www.scielo.br.

Dancey, C. P., \& Reidy, J. (2006). Estatística sem matemática para psicologia. Porto Alegre: Artmed/Bookman.

Felipe, T. A. (2006). Políticas públicas para a inserção da Libras na educação de surdos. Espaço: Informativo Técnico-Científico do INES, 25.

Franco, T., Druck, G. \& Seligmann-Silva, E. (2010). As novas relações de trabalho, o desgaste mental do trabalhador e os transtornos mentais no trabalho precarizado. Revista Brasileira de Saúde Ocupacional, 35(122), 229-248.

Freeman, R., Carbim, C. \& Boese, R. J. (1999). Um guia para todos os que lidam com crianças surdas. Santos: Brasília.

Klein, M. (1998). Surdez um olhar sobre a diferença. Porto Alegre: Medição.

Locke, E. A. (1976). The nature and causes of job satisfaction. Em Dunnette, M. D. (Org.). Handbook of industrial and organizational psychology (pp. 12971350). Chicago: Rand McNally College Publishing Company, management guide. Windsor: NFER -Nelson, 1988.

Margis, R., Picon, P., Cosner, A. F. \& Silveira, R. O. (2003). Relação entre estressores, estresse e ansiedade. Revista de Psiquiatria do Rio Grande do Sul, 25(1), 65-74.

Martins, M. C. F. (1984) Satisfação no trabalho: elaboração de instrumento e variáveis que afetam a satisfação Dissertação de Mestrado. Universidade de Brasília - Instituto de Psicologia, Brasília.

Martins, M. C. F., \& Santos, G. E. (2006). Adaptação e Validação de construto da Escala de Satisfação no Trabalho. Psico-USF, 11(2), 195-205.

Mendes, E. G., Nunes, L. R. D. P., Ferreira , J. R., \& Silveira, L. C. (2004). Estado da arte das pesquisas sobre profissionalização do portador de deficiência. Temas em Psicologia da SBP, 12(2), 105-118.

Miguel, F. K. \& Noronha, A. P. P. (2007) Estudos dos Parâmetros Psicrométricos da Escala de Vulnerabilidade ao Estresse no Trabalho. Evaluar, 7, 1-18

Nambu, T. S. (2003). Construindo um mercado de trabalho inclusivo: guia prático para profissionais de recursos humanos. São Paulo: SORRI-BRASIL; Brasília: CORDE. 
Noronha, A. P. P. \& Fernandes, D. C. (2007). Estresse laboral e raciocínio inferencial: Um estudo correlacional. Psicologia: Ciência e Profissão, 27(4), 596-607.

O'Reilly, C. A. (1990). Organizational behavior: Where we've been, where we're going. Annual Review of Psychology, 2, 427-58.

Omote, S. (1994). Deficiência e não-deficiência: recortes do mesmo tecido. Revista Brasileira de Educação Especial, 1(2), 65-73.

Paschoal, T. \& Tamayo, A. (2004). Validação da Escala de Estresse no Trabalho. Estudos de Psicologia, 9(1), 45-52.

Rebouças, D., Abelha L., Legay, L. F. \& Lovisi, G. M. (2008) O trabalho em saúde mental: um estudo de satisfação e impacto. Cadernos de Saúde Pública, 24(3), 624-632.

Ruviaro, M. F. S., \& Bardagi, M. P. (2010). Síndrome de burnout e satisfação no trabalho em profissionais da área de enfermagem do interior do RS. Barbarói, 33, 194-216.

Sassaki, R. K. (1997). Inclusão: constuindo uma sociedade para todos. Rio de Janeiro: WVA.

Selye, H. (1982). History and present status of the stress concept. Em: L. Goldberger, \& S. Breznitz (Orgs). Handbook of Stress. Theoretical and Clinical Aspects (pp. 7-17). London: Collier Macmillan Publishers.

Siqueira, M. M. M. (1995). Antecedentes de comportamento de cidadania organizacional: A análise de um modelo pós-cognitivo. Tese de Doutorado, Universidade de Brasília, Brasília.

Siqueira, M. M. (2008). Medidas do comportamento organizacional: ferramentas de diagnóstico de gestão. Porto Alegre: Artmed.

Sisto, F. F., Baptista, M. N., Noronha, A. P. P., \& Santos, A. A. A. (2007). Escala de vulnerabilidade a estressores no trabalho. São Paulo: Vetor Editora Psicopedagógica.

Sisto, F. F., Baptista, M. N., Santos, A. A. A., \& Noronha, A.P. P. (2008). Análise Fatorial da Escala de Vulnerabilidade ao Estresse no Trabalho (EVENT). Psicologia para América Latina, 15, 24-30.

Suehiro, A. C. B., Santos, A. A. A., Hatamoto, C. T., \& Cardoso, M. M. (2008). Vulnerabilidade ao estresse e satisfação no trabalho em profissionais do programa de saúde da família. Boletim de Psicologia, 58(129), 205-218. 
Taylor, F. (1947). Principles of scientific management. New York: Harper Brothers.

Violante, R. R., \& Leite, L. P. (2011). A empregabilidade das pessoas com deficiência: uma análise da inclusão social no mercado de trabalho do município de Bauru, SP. Cadernos de Psicologia Social do Trabalho, 14(1), 73-91.

Contato: rodolfo.ambiel@saofrancisco.edu.br

Recebido em: 01/03/2012

Revisado em: 08/05/2012

Aceito em: 05/06/2012 